OPEN ACCESS

Edited by:

Kyung Tae,

Hanyang University, South Korea

Reviewed by:

Tzu-Yen Huang,

Kaohsiung Medical University, Taiwan

Leong-Perng Chan,

Kaohsiung Medical University Hospital, Taiwan

Ming Xu,

The First Affiliated Hospital of Sun Yat-Sen University, China

Jia Joanna Wang,

Kaohsiung Medical University Hospital, Taiwan

${ }^{*}$ Correspondence:

Yukun LuO

lyk301@163.com

Specialty section: This article was submitted to

Thyroid Endocrinology,

a section of the journal

Frontiers in Endocrinology

Received: 11 September 2021 Accepted: 27 December 2021

Published: 17 February 2022

Citation:

Yan L, Zhang M, Li X, Li Y and Luo $Y$ (2022) A Nomogram to Predict Regrowth After Ultrasound-

Guided Radiofrequency Ablation for Benign Thyroid Nodules.

Front. Endocrinol. 12:774228. doi: 10.3389/fendo.2021.774228

\section{A Nomogram to Predict Regrowth After Ultrasound-Guided Radiofrequency Ablation for Benign Thyroid Nodules}

\author{
Lin Yan, Mingbo Zhang, Xinyang Li, YingYing Li and Yukun Luo* \\ Department of Ultrasound, First Medical Center, Chinese PLA General Hospital, Beijing, China
}

Objective: To develop and validate a nomogram to predict regrowth for patients with benign thyroid nodules undergoing radiofrequency ablation (RFA).

Methods: A total of 200 patients with 220 benign thyroid nodules who underwent RFA were included in this respective study. After RFA, patients were followed up at 1, 3, 6, and 12 months, and every 12 months thereafter. Regrowth was defined as an increase in nodule volume 50\% over the previously recorded smallest volume. A nomogram was developed based on the variables identified by multivariate logistic regression and the model performance was evaluated by discrimination(concordance index) and calibration curves.

Results: The incidence of regrowth was 13.64\% (30/220) after a mean follow-up period of $27.43 \pm 17.99$ months. Multivariate logistic regression revealed initial volume $(\mathrm{OR}=$ $1.047,95 \% \mathrm{Cl} 1.020-1.075)$, vascularity $(\mathrm{OR}=2.037,95 \% \mathrm{Cl} 1.218-3.404)$, and location close to critical structure $(\mathrm{OR}=4.713,95 \% \mathrm{Cl} 1.817-12.223)$ were independent factors associated with regrowth. The prognostic nomogram incorporating these three factors achieved good calibration and discriminatory abilities with a concordance index of 0.779 (95\% Cl 0.686-0.872).

Conclusions: A prognostic nomogram was successfully developed to predict nodule regrowth after RFA, which might guide physician in stratifying patients and provide precise guidance for individualized treatment protocols.

Keywords: ablation techniques, radiofrequency ablation, ultrasonography, nomograms, thyroid nodule

\section{INTRODUCTION}

Thyroid nodules are a common finding, present in up to $65 \%$ of the general population (1). Most nodules are benign and asymptomatic; however, a minority may require treatment because of the compressive symptomatic or cosmetic problems. Surgery is the standard treatment, which can remove a nodule completely at the cost of potential risk of complications, scar formation, and damage to normal thyroid parenchyma $(2,3)$. Thus, there has been a growing interest in developing 
the minimally invasive treatment that is much safer and allows gland retention for the treatment of benign thyroid nodules.

Ultrasound (US)-guided thermal ablation techniques, namely, radiofrequency ablation (RFA), microwave ablation (MWA), and laser ablation (LA) have been recommended as alternatives to surgery for benign thyroid nodules (3-7). These thermal ablation techniques have been successfully applied in treating patients with benign thyroid nodules (8-16). A recent meta-analysis included 24 studies on ablation and found that the volume reduction rates (VRR) at 6, 12,24, and 36 months during the follow-up period were $60,66,62$, and 53\%, respectively (17). Moreover, the major complications rate was only $1.3 \%$ without life-threatening adverse events (18). Although satisfactory results were achieved, several studies found that approximately $5.6-38 \%$ of the treated nodule occurred regrowth after 2 to 3 years after ablation (19-23). Regrowth was defined as $\geq 50 \%$ volume increase compared to the previously recorded smallest volume (24). It usually occurred from the untreated peripheral area and additional ablation might be beneficial $(19,20,22,25,26)$. Therefore, early evaluation of the risk of regrowth after ablation for benign thyroid nodules was essential for the treatment planning and follow-up strategy. Previous studies found that when dividing the total volume of ablated nodule after ablation into the ablated volume (Va) and the incompletely vital volume $(\mathrm{Vv})$, an increased $\mathrm{Vv}$ could be an early sign of nodule regrowth $(20,27)$. Several other predictors were also reported to be associated with regrowth, namely, the 12-month VRR (23), energy applied per volume (EPV) (21), and residual vital ratio (RVR) (27). Because they could be only obtained during the follow-up after ablation, none of them could be used as an early predictor for nodule regrowth. Negro et al. (28) recent developed a machine learning algorithm to discriminate nodules with a VRR $>50 \%$ at 12 months after RFA to identify the best candidates for effective treatment in one single session. However, accurate prediction of nodule regrowth after ablation is still lacking. A nomogram has been considered to be evidence-based, individualized and accurate in risk estimation, which has been applied to various malignancies (29-32). It has also been used to predict the clinical outcomes of patients with hepatocellular carcinoma who underwent thermal ablation (33-36). However, to our best knowledge, a prognostic nomogram for benign thyroid nodules following RFA has not yet been reported.

Therefore, the purpose of this study was to construct a nomogram to predict regrowth for patients with benign thyroid nodules undergoing RFA.

\section{MATERIALS AND METHODS}

Approval for this retrospective study was obtained from the Institutional Review Board of our institution (No. S2019-211101). Written information consent was obtained from all the patients prior to RFA procedure.

\section{Patients}

The study inclusion criteria were as follows: (1) nodules confirmed as benign via two separated fine-needle aspiration or core-needle biopsy; (2) nodules without suspicious malignant features on US examination, namely, very hypoechoic, irregular margin, taller than wide, microcalcification, evidence of extrathyroidal extension, and suspicious cervical lymph node; (3) patients with $\operatorname{solid}(\leq 10 \%$ of fluid component) or predominantly solid nodules (11-50\% of fluid component) (24); (4) patients who were complained about cosmetic, symptomatic problems or rapid growth; (5) serum thyroid hormone and thyrotropin levels within normal ranges; (6) patients who were refusal or ineligibility for surgical treatment; and (7) follow-up time was larger than 6 months. Exclusion criteria were: (1) malignancy findings or follicular neoplasm by biopsy; (2) nodules with benign result by biopsy had suspicious of malignancy on US examination; and (3) follow-up time was less than 6 months.

We retrospectively searched the database for patients who would be eligible for this study and selected the period from August 2014 to December 2018. After the exclusion of patients, 200 patients with 220 benign thyroid nodules with full medical records were included in this study.

\section{Pre-Ablation Assessment}

US were performed using a Siemens Acuson Sequoia 512 Ultrasound System (Siemens, Mountain View, CA, USA) or a Philips iU22 Ultrasound System (Philips Healthcare, Bothell, WA) or a Mindray M9 Ultrasound System (Mindray, Shenzhen, China). Before RFA, patients underwent US, biopsy, clinical evaluation, and also the laboratory examination. The nodule volume was calculated by ellipsoid formula: $\mathrm{V}=\pi \mathrm{abc} / 6(\mathrm{~V}$, nodule volume; $a$, the largest nodule diameter; $b$ and $c$, the other two perpendicular diameters of the nodule). Nodule location was classified as normal location and close to critical structures (less than $2 \mathrm{~mm}$ ), namely, trachea, cervical carotid artery, jugular vein, esophagus and recurrent laryngeal nerve. According to the component, the nodules were categorized as solid $(\leq 10 \%$ of fluid component) and predominantly solid (11-50\% of fluid component) (24). Nodule vascularity was classified using a 1-4 grade scale (4): grade 1, no vascularity; grade 2, peripheral vascularity; grade 3, intra-nodular vascularity $<50 \%$; and grade 4 , intra-nodular vascularity $\geq 50 \%$. The nodule-related symptom score was self-measured by the patient using a $10-\mathrm{cm}$ visual analogue scale (grades $0-10$ ) (4). The cosmetic score was evaluated by the physician as follows: 1 , no palpable mass; 2 , no cosmetic problem but palpable mass; 3 , a cosmetic problem on swallowing only; and 4, a readily detected cosmetic problem) (4). The laboratory examination included a complete blood count, thyroid function tests, and also the blood coagulation tests.

\section{Ablation Procedure}

One experienced US physician performed all RFA procedures using a bipolar RFA generator (CelonLabPOWER, Olympus Surgical Technologies Europe) and an 18-gauge bipolar RF electrodes with $0.9 \mathrm{~cm}$ active tip (CelonProSurge micro 100T09, Olympus Surgical Technologies Europe).

Patients were in supine position with their necks extended. RFA was performed using the trans-isthmic approach and moving-shot technique after local anesthesia with $1 \%$ 
lidocaine. If the distance between the nodule and surrounding critical structures was $<5 \mathrm{~mm}$, hydrodissection technique was performed with injection of normal saline to prevent thermal injury. The output RFA power was 3-9 W. Contrast-enhanced ultrasound (CEUS) was conducted immediately after the RFA procedure to evaluate the ablation area. CEUS was performed after bolus injection of SonoVue $(2.4 \mathrm{ml}$, Bracco), followed by a 5 $\mathrm{ml}$ of normal saline flush. If any enhancement existed in the treated nodule, a supplementary ablation was performed. The patients were observed for 1-2 $\mathrm{h}$ to check for possible adverse events or side effects.

\section{Post-Ablation Evaluation}

After ablation, patients were followed up at 1, 3, 6, and 12 months and every 12 months thereafter and the volume, VRR, cosmetic and symptom scores were evaluated. VRR was calculated as follows: $\operatorname{VRR}=[($ initial volume - final volume $) \times$ $100 \%$ ] / initial volume (24). A >50\% volume reduction at 12 months after ablation was defined as technical efficacy (24). Regrowth was defined as an increase in total volume 50\% over the previously recorded smallest volume (24). According to the detection of regrowth, the nodules were divided into nonregrowth group and regrowth group. To exclude neoplastic transformation, CNB was performed to all the regrowth nodules, which was performed by a disposable $1.5-$ or $2.2-\mathrm{cm}$ excursion, 20-gauge double-action spring-loaded needle (BARD Magumn, Bard Peripheral Vascular, Inc.) to the vital area of the nodule.

\section{Statistical Analysis}

Continuous variables were reported as mean \pm SD and categorical variables were expressed as numbers with percentages. Mann-Whitney $U$ test were used to compare volume, VRR, symptom and cosmetic scores between the two groups. Univariate and multivariate logistic regression analysis of independent factors influencing regrowth were assessed, and the odds ratios (OR) with 95\% confidence intervals (CI) were reported. A nomogram incorporated these independent factors was constructed to predict the probability of regrowth after RFA. The model performance was evaluated by discrimination and calibration (29). The discrimination of the nomogram was evaluated by the concordance index (C-index), which was equivalent to the area under the receiver operating characteristic curve. The value of the $\mathrm{C}$-index varied between 0.5 and 1.0, with 1.0 indicating the perfect ability to correctly discriminate outcomes, and 0.5 indicating a random chance. Model validation was performed using bootstrap validation method with 1,000 resamples to quantify the overfitting of modeling strategy and predict future performance of the model (29). Calibration was evaluated using a calibration curve, which was a graphic representation of the relationship between the observed outcome frequencies and the predicted probabilities, with 1,000 bootstrap resamples of the study group. In a wellcalibrated model, the predictions should fall on a 45-degree diagonal line. Statistical analyses were performed by using SPSS statistical software(Version 25.0) and R software version
3.6.2 (R Foundation for Statistical Computing). A two-sided $P<0.05$ was considered as statistically significant.

\section{RESULTS}

In this study, 200 patients (176 females, 24 males, mean age $46.02 \pm$ 11.95 years) with 220 benign thyroid nodules were enrolled (Table 1). Among these 200 patients, 183 patients had 1 nodule, 14 patients had 2 nodules and 3 patients had 3 nodules. The initial volume was $10.30 \pm 13.41 \mathrm{ml}$ and the largest diameter was $3.00 \pm$ $1.37 \mathrm{~cm}$. During RFA, the mean power of was $6.83 \pm 3.11 \mathrm{~W}$. The mean energy was 2,639.32 $\pm 2,173.70 \mathrm{~J}$ and the mean EPV was $648.04 \pm 678.55 \mathrm{~J} / \mathrm{ml}$.

The mean follow-up time was $27.43 \pm 17.99$ months. The mean VRR was $88.82 \pm 11.98 \%$ and the technical efficacy was $96.82 \%(213 / 220)$. Symptom score significantly decreased from $2.72 \pm 2.15$ to $0.95 \pm 1.18(P<0.001)$. Cosmetic score significantly decreased from $2.45 \pm 1.22$ to $1.34 \pm 0.58(P<0.001)$.

Regrowth was observed in 30 out 220 nodules (13.64\%), which all occurred in the untreated peripheral area. The mean timing of regrowth was at $22.40 \pm 12.10$ months after RFA. All the regrowth nodules underwent additional RFA. The changes of volume and VRR in the two groups are present in Table 2. In the first 12 months, VRR in the two groups were nonsignificant (all $P>0.05)$. However, at 24 months after RFA, VRR in the nonregrowth group were significantly larger than that in the regrowth group $(90.39 \pm 11.23 \%$ vs $78.65 \pm 12.24 \%, P=0.002)$ (Figure 1). A total of 7 nodules had volume reduction less than $50 \%$ at 12 months. Five nodules were in the regrowth group and 2 in the non-regrowth group $(16.67 \%$ vs $1.05 \%, P<0.001)$. Representative cases in the two groups are shown in Figures 2, 3.

The comparison of the two groups is shown in Table 3. The initial volume $(19.33 \pm 19.79 \mathrm{ml}$ vs $8.88 \pm 11.54 \mathrm{ml}, P<0.001)$ and vascularity ( $3.0 \pm 0.8$ vs $2.4 \pm 0.9, P=0.002)$ in the regrowth group were significantly larger than those in the non-regrowth group. In the regrowth group, $33.67 \%(11 / 30)$ nodules were located close to the critical structure, while in the non-regrowth group $17.37 \%(33 / 190)$ of the nodules were close to the critical structure $(P=0.017)$. The energy and EPV in the regrowth group and non-regrowth group were nonsignificant $(3,369.66 \pm 2,281.05 \mathrm{~J}$ vs $2,527.84 \pm 2,141.21 \mathrm{~J}, P=$ $0.070 ; 440.49 \pm 684.83 \mathrm{~J} / \mathrm{ml}$ vs $679.72 \pm 673.78 \mathrm{~J} / \mathrm{ml}, P=0.085)$.

TABLE 1 | Clinical characteristics.

\begin{tabular}{lc}
\hline Characteristics & Data \\
\hline No. of patients/nodules & $200 / 220$ \\
Age (years) & $46.02 \pm 11.95$ \\
Sex (F/M) & $176 / 24(88.0 / 12.0)$ \\
Component & \\
$\quad$ Solid/ Predominately solid & $155 / 65(70.5 / 20.5)$ \\
Location & \\
$\quad$ Right lobe/Left lobe/lsthmus & $114 / 104 / 2(51.82 / 47.27 / 0.91)$ \\
Largest diameter (cm) & $3.00 \pm 1.37$ \\
Initial volume (ml) & $10.30 \pm 13.41$ \\
Vascularity & $2.5 \pm 0.9$ \\
\hline
\end{tabular}

Data are presented as mean $\pm S D$ or number of nodules (percentages). 
TABLE 2 | Changes of volume and VRR at each follow-up period after RFA.

\begin{tabular}{|c|c|c|c|c|c|c|c|c|}
\hline \multirow[t]{2}{*}{ Time (months) } & \multicolumn{4}{|c|}{ Volume (ml) } & \multicolumn{4}{|c|}{ VRR (\%) } \\
\hline & Total & Non-regrowth group & Regrowth group & $P$-value & Total & Non-regrowth group & Regrowth group & $P$-value \\
\hline 1 & $4.89 \pm 6.19$ & $4.60 \pm 6.22$ & $6.86 \pm 5.75$ & 0.007 & $48.62 \pm 19.63$ & $48.26 \pm 18.79$ & $51.12 \pm 25.03$ & 0.350 \\
\hline 3 & $2.72 \pm 3.79$ & $2.44 \pm 3.54$ & $4.54 \pm 4.82$ & 0.023 & $69.89 \pm 16.61$ & $69.59 \pm 16.50$ & $71.83 \pm 17.60$ & 0.394 \\
\hline 6 & $2.60 \pm 4.13$ & $2.21 \pm 3.44$ & $4.65 \pm 6.37$ & 0.015 & $79.42 \pm 13.44$ & $79.22 \pm 13.58$ & $80.46 \pm 12.95$ & 0.595 \\
\hline 12 & $2.06 \pm 4.07$ & $1.76 \pm 3.36$ & $4.42 \pm 7.39$ & 0.031 & $84.38 \pm 14.07$ & $84.92 \pm 13.04$ & $80.08 \pm 20.47$ & 0.489 \\
\hline 24 & $1.90 \pm 3.81$ & $1.48 \pm 3.13$ & $4.60 \pm 6.31$ & 0.005 & $88.82 \pm 11.98$ & $90.39 \pm 11.23$ & $78.65 \pm 12.24$ & 0.002 \\
\hline
\end{tabular}

Data are presented as mean $\pm S D$.

Multivariate logistic regression revealed initial volume $(\mathrm{OR}=$ $1.047,95 \%$ CI $1.020-1.075)$, vascularity (OR $=2.037,95 \%$ CI $1.218-$ $3.404)$ and location close to critical structure $(\mathrm{OR}=4.713,95 \% \mathrm{CI}$ 1.817-12.223) were independent factors associated with regrowth (Table 4). A nomogram based on these three independent factors to predict nodule regrowth after RFA was constructed (Figure 4). Each factor was allocated a predicting score, and the sum of three scores was located on the total points axis, suggesting the prediction of regrowth probabilities. Higher total points were associated with a higher regrowth probability during the followup. The discriminative ability of the model for regrowth was assessed using the C-index, which was 0.779 (95\%CI 0.686-0.872) (Figure 5). The accuracy of the model and potential model overfit were assessed by bootstrap validation with 1,000 re-samplings. The calibration curves graphically showed good agreement between the actual and nomogram-predicted regrowth (Figure 6).

No major complications occurred during or after RFA. Eighteen patients $(9.00 \%)$ had local pain or discomfort and resolved spontaneously within 1 week.

\section{DISCUSSION}

No prediction model for regrowth after thermal ablation for benign thyroid nodules has been reported to date. This study

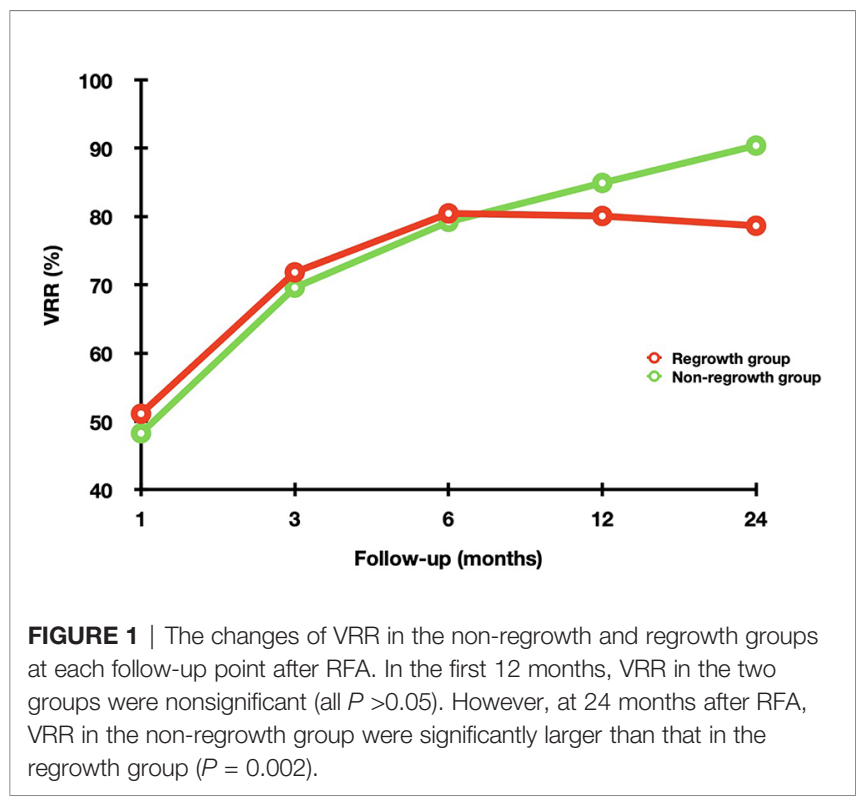

developed and validated a nomogram to predict regrowth after RFA for benign thyroid nodules. The $\mathrm{C}$-index of this nomogram was 0.779 with a good calibration, which suggested that the nomogram could successfully stratified patients before treatment according to their risk of regrowth and yielded excellent performance. This model would be helpful to guide decisionmaking and provide individualized ablation management.

As alternatives to surgery for benign thyroid nodules by guidelines (4-6), the ablation efficacy has attracted much attention. The volume reduction after ablation was significant with a low rate of complications $(17,18,21)$. However, the effective treatment of ablation should be sustainable for longer follow-up period. Some studies showed a tendency for the nodules to regrow during the follow-up and the reported incidence of regrowth after RFA, MWA and LA was 5.6-24.1\% (19-21), $14.55 \%(22)$ and $37.8-38 \%(21,23)$, respectively. Several predictors were reported to be associated with regrowth, but the optimal prediction of regrowth was still unclear. Sim et al. (20) found that the total volume of nodule after ablation could be divided into $\mathrm{Va}$ and $\mathrm{Vv}$. The results found that $\mathrm{Vv}$ increase which was defined as a more than $50 \%$ increase compared to the previously reported smallest $\mathrm{Vv}$, occurred earlier than regrowth and might be used as an early sign (20). However, Vv increase was observed at $27.5 \pm 18.5$ months after RFA, making its predictive value limited. Negro et al. (23) reported that a 12 month VRR $<50 \%$ increased the risk of regrowth after LA with an OR of 11.7 (95\%CI 4.2-32.2). However, compared with LA, the efficacy of RFA or MWA seemed to be much better with a higher volume reduction $(17,37-40)$ and a lower regrowth rate (19-23). The predictive value of 12-month VRR of these two ablation techniques still needed further investigation. Furthermore, whether EPV could affect ablation efficacy or regrowth remained controversial. A higher EPV was found to be associated with the efficacy of ablation $(21,41,42)$. Wang et al. (22) found that EPV in the non-regrowth group was significantly larger than that in the regrowth group after MWA. However, contradictory results were also observed. Negro et al. (28) found that EPV was significantly larger in the nodules with 12 -month VRR $<50 \%$ than that in nodules with 12 -month $\operatorname{VRR} \geq 50 \%$, indicating the former were not treated with an insufficient amount of energy. In this study, although EPV in the non-regrowth group were larger than that in the regrowth group, no significant differences were found. The reason of different results might be related to the diverse thermal ablation techniques, nodule structure and volume, which could lead to different production and distribution of thermal energy (21), making the predict value of EPV still uncertain. 

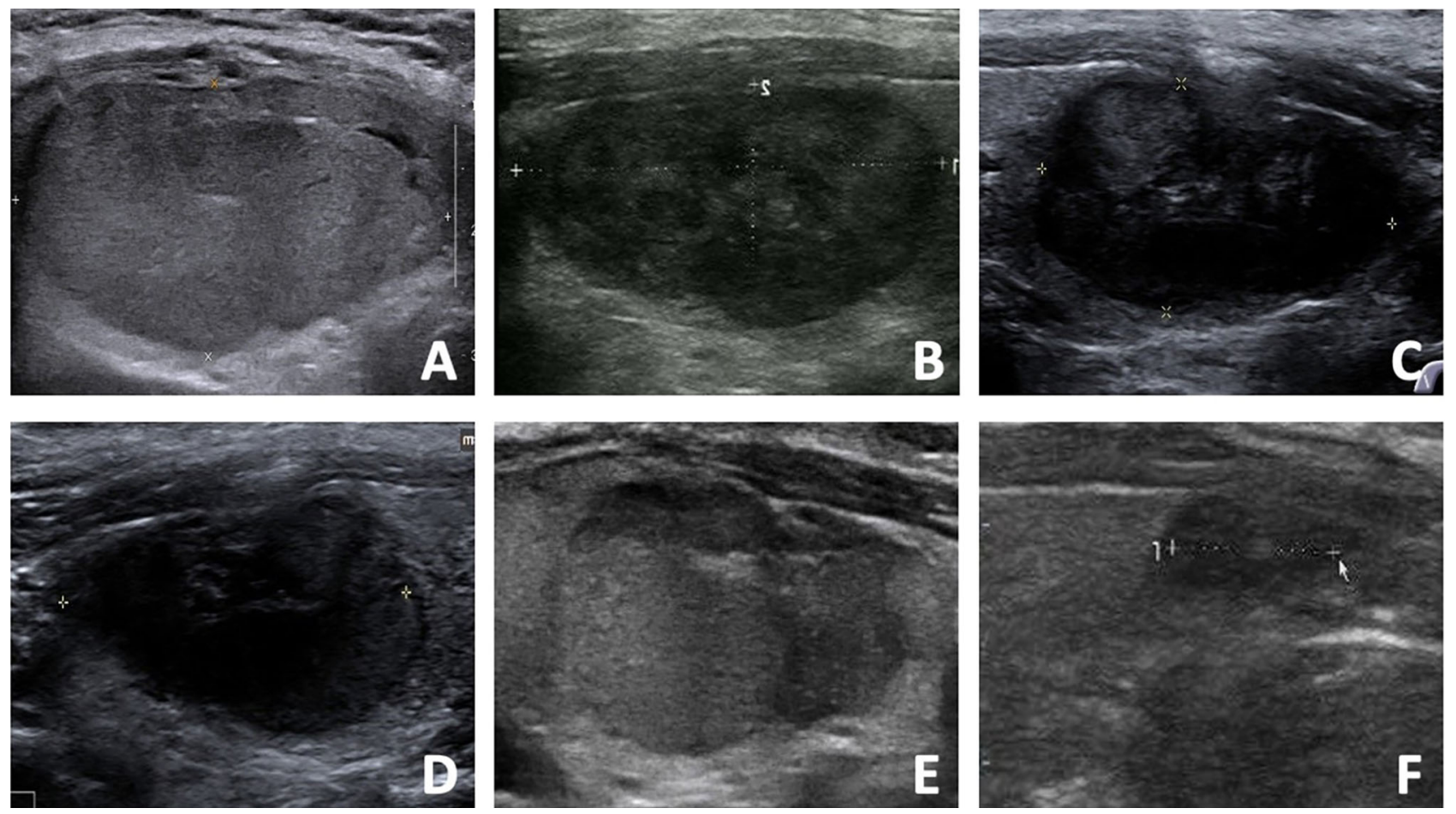

FIGURE 2 | The US images of a 48- year-old male with a benign thyroid nodule in the regrowth group. (A) The initial volume of nodule was $22.27 \mathrm{ml}$ before RFA. The nodule was located in the dangerous location, and the vascularity was grade 4 . The risk of regrowth by the nomogram was $67 \%$. (B) At 1 month after RFA, the volume was $5.28 \mathrm{ml}$ and VRR was $76.29 \%$. (C) At 3 months after RFA, the volume was $3.48 \mathrm{ml}$ and VRR was $84.37 \%$. (D) At 6 months after RFA, the volume was $2.89 \mathrm{ml}$ and VRR was $87.02 \%$. (E) At 12 months after RFA, the US showed nodule regrowth and the volume was $4.34 \mathrm{ml}$ and VRR was $80.51 \%$. Additional RFA was performed. (F) At 6 months after additional RFA, the volume was $1.65 \mathrm{ml}$ and VRR was $92.59 \%$.

CLT, also known as Hashimoto thyroiditis, is an autoimmune disease that destroys thyroid cells by cell and antibody-mediated immune process. The pathology of the disease involves the formation of antithyroid antibodies that attack the thyroid tissue, causing widespread lymphocyte infiltration, fibrosis, and parenchymal atrophy of the thyroid tissue. This study found that CLT was not an independent factor related to regrowth by multivariate analysis. It was consistent with previous study (43), which reported that the recovery after RFA in patients with PTMC + CLT was similar to that in patients with only PTMC. It suggested that although the thyroid parenchyma in the case of CLT was already infiltrated by diffuse chronic inflammatory cells, it did not influence the clinical outcomes of RFA for benign thyroid nodules.

In this study, a nomogram incorporated three easily identifiable variables was developed to predict nodule regrowth after RFA. The performance was well supported by the C-index of 0.779 and the calibration curves demonstrating the optimal agreements between prediction and actual observation, which guaranteed the repeatability and reliability of the established nomogram. To the best of our knowledge, it was the first nomogram to predict nodule regrowth after ablation, which could be helpful to improve the patient-physician communication, decision-making and individualized treatment management. Though this easy-to-use scoring system, physicians could perform an individualized prediction before ablation to identify patients at different risk of regrowth. Patients with a high score had an increased probability of nodule regrowth and were potential candidates for additional treatment. Therefore, these patients needed to be provided appropriate ablation strategy and intensive follow-up management or recommended surgery if they do not want multiple sessions.

So far, no consensus about the specific indication or optimal timing of additional ablation for benign thyroid nodules was achieved (43). In most studies, additional ablation was usually determined based on clinical evaluation findings, namely, incompletely symptom resolution, or unsatisfactory volume reduction, or regrowth (4). Sim et al. (20) suggested that additional ablation might be performed after the detection of $\mathrm{Vv}$ increase because it was important to identify regrowth. A recent study compared the efficacy of additional RFA after different indications. Additional RFA performed after $\mathrm{Vv}$ increased revealed superior efficacy, including a significant greater volume reduction improvement of cosmetic and symptom scores (44). It was because that the residual volume was markedly larger when $\mathrm{Vv}$ increase was observed. Additional ablation could be applied to a larger residual zone and achieve 

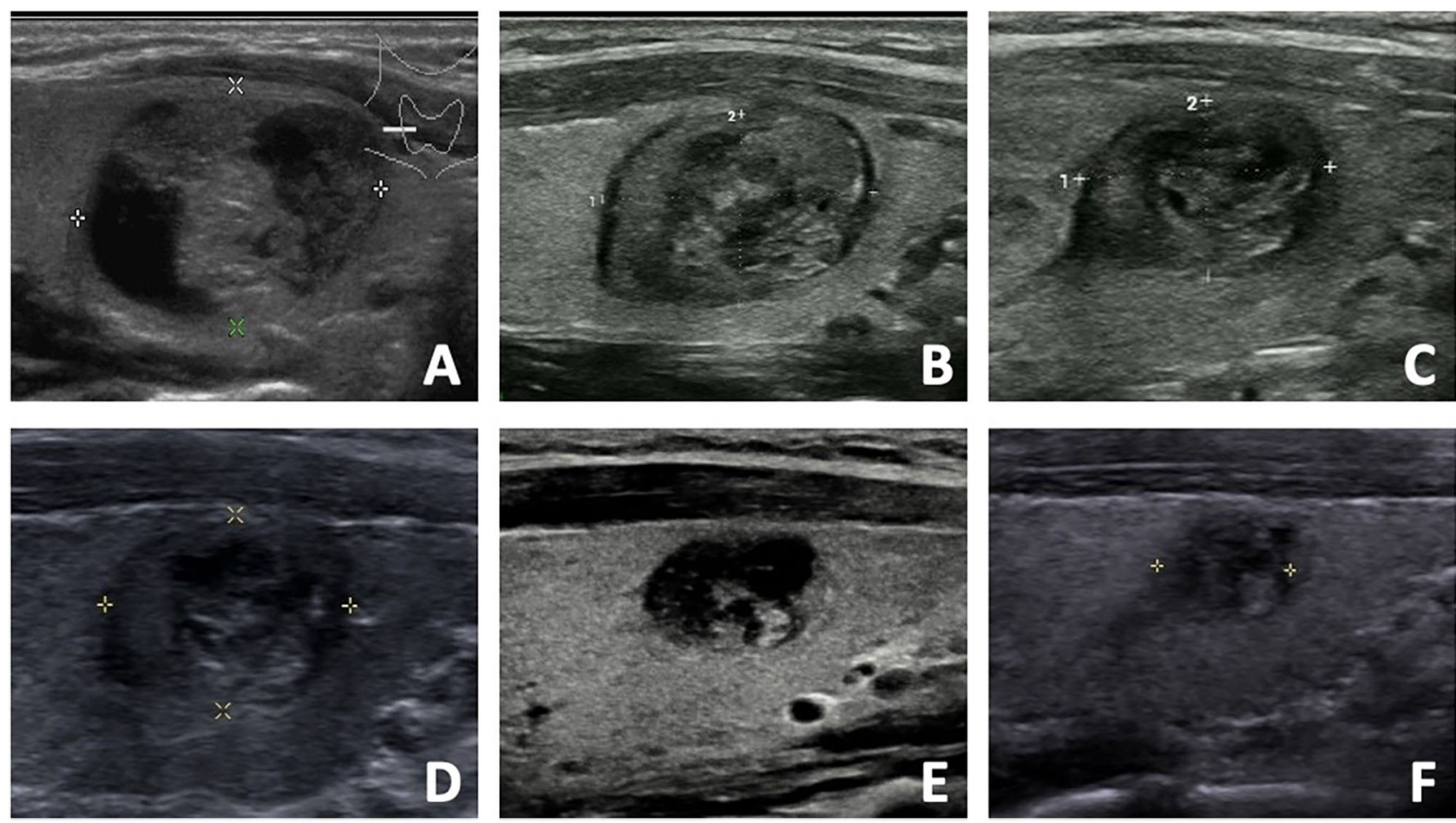

FIGURE 3 | The US images of a 33- year-old female with a benign thyroid nodule in the non-regrowth group. (A) The initial volume of nodule was 5.32 ml before RFA. The nodule was located in the normal location, and the vascularity was grade 1. The risk of regrowth by the nomogram was $2.1 \%$. (B) At 1 month after RFA, the volume was 1.58 $\mathrm{ml}$ and VRR was $70.30 \%$. (C) At 3 months after RFA, the volume was $0.88 \mathrm{ml}$ and VRR was $83.46 \%$. (D) At 6 months after RFA, the volume was $0.76 \mathrm{ml}$ and VRR was 85.71\%. (E) At 12 months after RFA, the volume was $0.32 \mathrm{ml}$ and VRR was $93.98 \%$. (F) At 18 months after RFA, the volume was $0.18 \mathrm{ml}$ and VRR was $96.62 \%$.

substantial incremental volume reduction. Therefore, to maximize the efficacy of ablation, additional RFA could be considered after the detection of $\mathrm{Vv}$ increase.

This study found that initial volume, location close to critical structure and vascularity were independent factors related to regrowth. Initial volume has been recognized as an important factor for regrowth $(19,20,43)$. It might be difficult to ablate all the nodule margin of a large nodule by a single session, leading to incomplete treatment and subsequent regrowth. Location was another factor associated with regrowth (22). The neck was relatively narrow, where contained many critical structures. As a result, complications might be inevitable when a nodule located close to the critical structures. For hyper-vascular nodules, the possibility of regrowth also existed (22). The vasculature in the thyroid nodules could cause heat-sink effect, which decreased the ablation efficacy and induced regrowth (45). To obtain complete ablation, several techniques have been recommended, namely, the moving shot technique, the hydrodissection

TABLE 3 | The comparison between the non-regrowth and regrowth groups.

\begin{tabular}{|c|c|c|c|c|}
\hline Characteristics & Non-regrowth group & Regrowth group & OR $(95 \% \mathrm{Cl})$ & $P$-value \\
\hline \multicolumn{5}{|l|}{ Pre-treated variables } \\
\hline Age (years) & $46.84 \pm 11.98$ & $42.55 \pm 11.35$ & $0.970(0.938-1.003)$ & 0.074 \\
\hline Female & $163(85.8)$ & $24(80.0)$ & $0.663(0.248-1.771)$ & 0.412 \\
\hline Solid & $137(72.11)$ & $18(60.00)$ & $0.580(0.262-1.287)$ & 0.180 \\
\hline Initial volume (ml) & $8.88 \pm 11.54$ & $19.33 \pm 19.79$ & $1.043(1.019-1.068)$ & $<0.001$ \\
\hline Location close to critical structure & $33(17.37)$ & $11(33.67)$ & 2.754 (1.199-6.330) & 0.017 \\
\hline Hashimoto's thyroiditis & $34(17.89)$ & $3(10)$ & $0.510(0.146-1.778)$ & 0.290 \\
\hline Vascularity & $2.4 \pm 0.9$ & $3.0 \pm 0.8$ & $2.161(1.312-3.561)$ & 0.002 \\
\hline \multicolumn{5}{|l|}{ Ablate-related variables } \\
\hline Power (W) & $6.88 \pm 3.25$ & $6.50 \pm 2.01$ & $0.957(0.831-1.101)$ & 0.535 \\
\hline Energy $(\mathrm{J})$ & $2,527.84 \pm 2,141.21$ & $3,369.66 \pm 2,281.05$ & $1.148(0.989-1.333)$ & 0.070 \\
\hline EPV $(\mathrm{J} / \mathrm{ml})$ & $679.72 \pm 673.78$ & $440.49 \pm 684.83$ & $0.999(0.998-1.000)$ & 0.085 \\
\hline
\end{tabular}

Data are presented as mean $\pm S D$ or number of tumors (percentages).

EPV, Energy per volume. 
TABLE 4 | Multivariate logistic regression analysis of regrowth.

\begin{tabular}{lrr}
\hline Characteristics & OR & 95\% Cl \\
\hline Initial volume $(\mathrm{ml})$ & 1.047 & $1.020-1.075$ \\
Location close to critical structure & 4.713 & $<0.001$ \\
Vascularity & 2.037 & 0.001 \\
\end{tabular}

Points

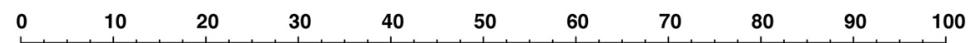

Initial volume

\begin{tabular}{lllllllll}
\hline 0 & 10 & 20 & 30 & 40 & 50 & 60 & 70 & 80
\end{tabular}

Location

Location close to critical structure

Normal

Vascularity

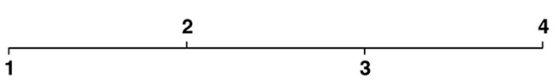

Total Points

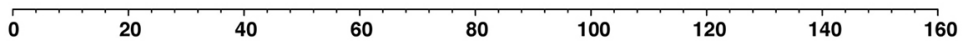

Regrowth after RFA

$\begin{array}{lllllllll}0.05 & 0.1 & 0.2 & 0.3 & 0.4 & 0.5 & 0.6 & 0.7 & 0.8\end{array}$

FIGURE 4 | The nomogram for predicting regrowth after RFA for benign thyroid nodules. A nomogram based on initial volume, vascularity and location was constructed.

technique, and vascular ablation techniques (4). The moving shot technique has been suggested as a suitable method for the peripheral ablation. This technique could treat the nodule

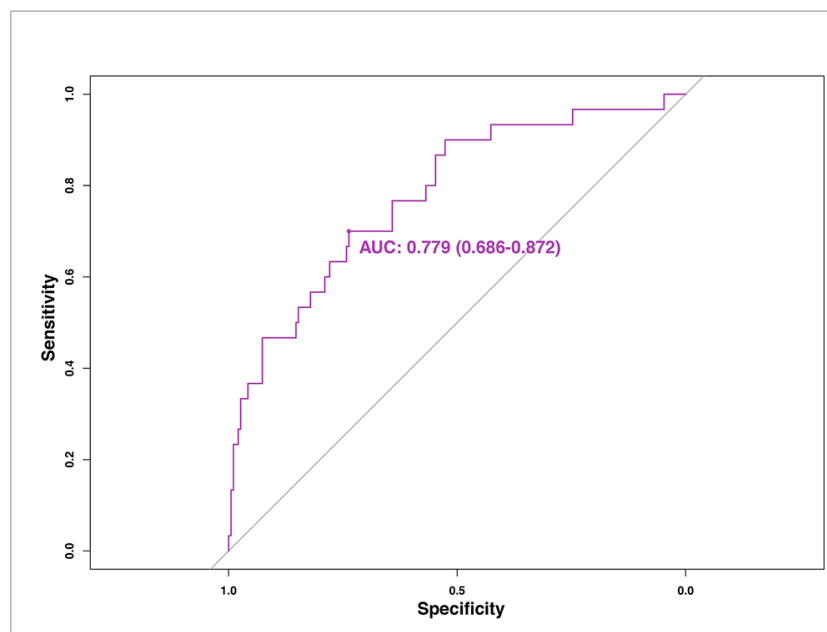

FIGURE 5 | The ROC of the nomogram for predicting regrowth in patients with benign thyroid nodules after RFA. AUC for the nomogram to predict regrowth was $0.779(95 \% \mathrm{Cl} 0.686-0.872)$. completely and safely by dividing it into multiple small ablation units (45). Moreover, the hydrodissection technique was effective to separate the nodule from the adjacent critical structures $(45,46)$. By injecting the fluid into the naturally existed gap between the cervical anatomical structures, a protective thermal barrier was formed to minimize the complications and improve the efficacy. Additionally, vascular ablation techniques have been introduced as an advanced technique for hyper-vascular nodules (45). The main feeding artery was ablated first followed by the draining vein along the nodule margin, which could not only damage the blood vessels and decrease the heat-sink effect, but also prevent incomplete ablation of the peripheral area.

This study had some limitations. First, because of the retrospective nature, sample bias was existed. Moreover, some potentially variables were not available in the data set and thus could not be included in this nomogram, such as the experience of US physician, different ablation techniques and inflammatory response caused by ablation. A large prospective study is needed to further confirm the reliability of the nomogram. Second, although this nomogram was internally validated using bootstrap validation, the clinical use must be externally validated and evaluated. Third, the follow-up was relatively short. There were two peaks of nodule regrowth (20). First 


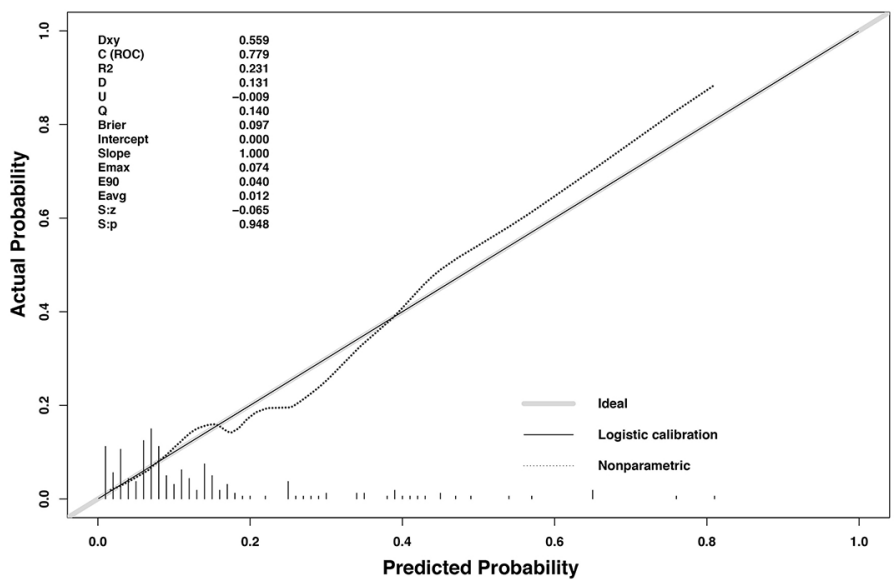

FIGURE 6 | The calibration curves for predicting regrowth in patients with benign thyroid nodules after RFA. The x-axis represents the nomogram predicted probability and the $y$-axis represents the actual probability. The calibration curves were close to the $45^{\circ}$ line. The diagonal dashed line indicates ideal prediction by a perfect model, and the solid line represents the predictive power of the nomogram. The closer the solid line is to the dotted line, the better is the predictive power of the model.

peak of regrowth ranged from 1 year to 2 or 3 years, and the second one appeared later than 5 years (20). The predictive value of this nomogram on the second peak of regrowth is still needed further investigation.

\section{CONCLUSION}

This study constructed a nomogram to predict regrowth in patients with benign thyroid nodules after RFA with good discrimination and calibration capabilities. The use of this prognostic nomogram may guide physician in stratifying patients and provide precise guidance for individualized treatment protocols.

\section{DATA AVAILABILITY STATEMENT}

The original contributions presented in the study are included in the article/supplementary files, further inquiries can be directed to the corresponding authors.

\section{REFERENCES}

1. Durante C, Grani G, Lamartina L, Filetti S, SJ M, Cooper DS. The Diagnosis and Management of Thyroid Nodules: A Review. JAMA (2018) 319(9):91424. doi: 10.1001/jama.2018.0898

2. Gharib H, Papini E, Paschke R, Duick DS, Valcavi R, Hegedus L, et al. American Association of Clinical Endocrinologists, Associazione Medici Endocrinologi, and European Thyroid Association Medical Guidelines for Clinical Practice for the Diagnosis and Management of Thyroid Nodules. J Endocrinol Invest (2010) 33(5 Suppl):1-50. doi: 10.4158/10024.GL

3. Haugen BR, Alexander EK, Bible KC, Doherty GM, Mandel SJ, Nikiforov YE, et al. 2015 American Thyroid Association Management Guidelines for Adult Patients With Thyroid Nodules and Differentiated Thyroid Cancer: The

\section{ETHICS STATEMENT}

The studies involving human participants were reviewed and approved by the Institutional Review Board of Chinese PLA General Hospital. The patients/participants provided their written informed consent to participate in this study.

\section{AUTHOR CONTRIBUTIONS}

YL interpreted the patient data and drafted the manuscript. LYK performed RFA procedure, conceived of the study and coordination. MBZ, XYL, YYL collected and analyzed the patient data. All authors contributed to the article and approved the submitted version.

\section{FUNDING}

This study is supported by the Popularization of Military Medical and Health Achievements (No. 21WKS09).

American Thyroid Association Guidelines Task Force on Thyroid Nodules and Differentiated Thyroid Cancer. Thyroid (2015) 26(1):1-133. doi: 10.1089/ thy. 2015.0020

4. Kim JH, Baek JH, Lim HK, Ahn HS, Baek SM, Choi YJ, et al. 2017 Thyroid Radiofrequency Ablation Guideline: Korean Society of Thyroid Radiology. Korean J Radiol (2018) 19(4):632-55. doi: 10.3348/kjr.2018.19.4.632

5. Papini E, Pacella CM, Solbiati LA, Achille G, Barbaro D, Bernardi S, et al. Minimally-Invasive Treatments for Benign Thyroid Nodules: A Delphi-Based Consensus Statement From the Italian Minimally-Invasive Treatments of the Thyroid (MITT) Group. Int J Hyperthermia (2019) 36(1):376-82. doi: 10.1080/02656736.2019.1575482

6. Dietrich CF, Müller T, Bojunga J, Dong Y, Mauri G, Radzina M, et al. Statement and Recommendations on Interventional Ultrasound as a Thyroid 
Diagnostic and Treatment Procedure. Ultrasound Med Biol (2018) 44(1):1436. doi: 10.1016/j.ultrasmedbio.2017.08.1889

7. Garberoglio R, Aliberti C, Appetecchia M, Attard M, Boccuzzi G, Boraso F, et al. Radiofrequency Ablation for Thyroid Nodules: Which Indications? The First Italian Opinion Statement. J Ultrasound (2015) 18(4):423-30. doi: 10.1007/s40477-015-0169-y

8. Deandrea M, Sung JY, Limone P, Mormile A, Garino F, Ragazzoni F, et al. Efficacy and Safety of Radiofrequency Ablation Versus Observation for Nonfunctioning Benign Thyroid Nodules: A Randomized Controlled International Collaborative Trial. Thyroid (2015) 25(8):890-6. doi: 10.1089/ thy.2015.0133

9. Cesareo R, Pasqualini V, Simeoni C, Sacchi M, Saralli E, Campagna G, et al. Prospective Study of Effectiveness of Ultrasound-Guided Radiofrequency Ablation Versus Control Group in Patients Affected by Benign Thyroid Nodules. J Clin Endocrinol Metab (2015) 100(2):460-6. doi: 10.1210/jc.20142186

10. Hamidi O, Callstrom MR, Lee RA, Dean D, Castro MR, Morris JC, et al. Outcomes of Radiofrequency Ablation Therapy for Large Benign Thyroid Nodules: A Mayo Clinic Case Series. Mayo Clin Proc (2018) 93(8):1018-25. doi: 10.1016/j.mayocp.2017.12.011

11. Radzina M, Cantisani V, Rauda M, Nielsen MB, Ewertsen C, D’Ambrosio F, et al. Update on the Role of Ultrasound Guided Radiofrequency Ablation for Thyroid Nodule Treatment. Int J Surg (2017) 41:S82-93. doi: 10.1016/ j.ijsu.2017.02.010

12. Rabuffi P, Spada A, Bosco D, Bruni A, Vagnarelli S, Ambrogi C, et al. Treatment of Thyroid Nodules With Radiofrequency: A 1-Year Follow-Up Experience. J Ultrasound (2019) 22(2):193-9. doi: 10.1007/s40477-019-00375-4

13. Kotewall N, Lang BHH. High-Intensity Focused Ultrasound Ablation as a Treatment for Benign Thyroid Diseases: The Present and Future. Ultrasonography (2019) 38(2):135-42. doi: 10.14366/usg.18040

14. Bernardi S, Dobrinja C, Carere A, Giudici F, Calabrò V, Zanconati F, et al. Patient Satisfaction After Thyroid RFA Versus Surgery for Benign Thyroid Nodules: A Telephone Survey. Int J Hyperthermia (2018) 35(1):150-8. doi: 10.1080/02656736.2018.1487590

15. Lang BHH, Woo YC, Chiu KW. Two-Year Efficacy of Single-Session HighIntensity Focused Ultrasound (HIFU) Ablation of Benign Thyroid Nodules. Eur Radiol (2019) 29(1):93-101. doi: 10.1007/s00330-018-5579-8

16. Lin WC, Kan NN, Chen HL, Luo SD, Tung YC, Chen WC, et al. Efficacy and Safety of Single-Session Radiofrequency Ablation for Benign Thyroid Nodules of Different Sizes: A Retrospective Study. Int J Hyperthermia (2020) 37 (1):1082-9. doi: 10.1080/02656736.2020.1782485

17. Trimboli P, Castellana M, Sconfienza LM, Virili C, Pescatori LC, Cesareo R, et al. Efficacy of Thermal Ablation in Benign Non-Functioning Solid Thyroid Nodule: A Systematic Review and Meta-Analysis. Endocrine (2019) 67(1):3543. doi: 10.1007/s12020-019-02019-3

18. Chung SR, Suh CH, Baek JH, Park HS, Choi YJ, Lee JH. Safety of Radiofrequency Ablation of Benign Thyroid Nodules and Recurrent Thyroid Cancers: A Systematic Review and Meta-Analysis. Int J Hyperthermia (2017) 33(8):920-30. doi: 10.1080/02656736.2017.1337936

19. Lim HK, Lee JH, Ha EJ, Sung JY, Kim JK, Baek JH. Radiofrequency Ablation of Benign Non-Functioning Thyroid Nodules: 4-Year Follow-Up Results for 111 Patients. Eur Radiol (2013) 23(4):1044-9. doi: 10.1007/s00330-012-2671-3

20. Sim JS, Baek JH, Lee J, Cho W, Jung SI. Radiofrequency Ablation of Benign Thyroid Nodules: Depicting Early Sign of Regrowth by Calculating Vital Volume. Int J Hyperthermia (2017) 33(8):905-10. doi: 10.1080/ 02656736.2017.1309083

21. Bernardi S, Giudici F, Cesareo R, Antonelli G, Cavallaro M, Deandrea M, et al. Five-Year Results of Radiofrequency and Laser Ablation of Benign Thyroid Nodules: A Multicenter Study From the Italian Minimally Invasive Treatments of the Thyroid Group. Thyroid (2020) 30(12):1759-70. doi: 10.1089 /thy.2020.0202

22. Wang B, Han ZY, Yu J, Cheng Z, Liu F, Yu XL, et al. Factors Related to Recurrence of the Benign Non-Functioning Thyroid Nodules After Percutaneous Microwave Ablation. Int J Hyperthermia (2017) 33(4):459-64. doi: 10.1080/02656736.2016.1274058

23. Negro R, Greco G, Deandrea M, Rucco M, Trimboli P. Twelve-Month Volume Reduction Ratio Predicts Regrowth and Time to Regrowth in Thyroid Nodules Submitted to Laser Ablation: A 5-Year Follow-Up
Retrospective Study. Korean J Radiol (2020) 21(6):764-72. doi: 10.3348/ kjr.2019.0798

24. Mauri G, Pacella CM, Papini E, Solbiati L, Goldberg SN, Ahmed M, et al. Image-Guided Thyroid Ablation: Proposal for Standardization of Terminology and Reporting Criteria. Thyroid (2019) 29(5):611-8. doi: 10.1089/thy.2018.0604

25. Dossing H, Bennedbaek FN, Hegedus L. Long-Term Outcome Following Interstitial Laser Photocoagulation of Benign Cold Thyroid Nodules. Eur J Endocrinol (2011) 165(1):123-8. doi: 10.1530/eje-11-0220

26. Valcavi R, Riganti F, Bertani A, Formisano D, Pacella CM. Percutaneous Laser Ablation of Cold Benign Thyroid Nodules: A 3-Year Follow-Up Study in 122 Patients. Thyroid (2010) 20(11):1253-61. doi: 10.1089/thy.2010.0189

27. Yan L, Luo Y, Xie F, Zhang M, Xiao J. Residual Vital Ratio: Predicting Regrowth After Radiofrequency Ablation for Benign Thyroid Nodules. Int $J$ Hyperthermia (2020) 37(1):1139-48. doi: 10.1080/02656736.2020.1825835

28. Negro R, Rucco M, Creanza A, Mormile A, Limone PP, Garberoglio R, et al. Machine Learning Prediction of Radiofrequency Thermal Ablation Efficacy: A New Option to Optimize Thyroid Nodule Selection. Eur Thyroid J (2020) 9 (4):205-12. doi: 10.1159/000504882

29. Iasonos A, Schrag D, Raj GV, Panageas KS. How to Build and Interpret a Nomogram for Cancer Prognosis. J Clin Oncol (2008) 26(8):1364-70. doi: $10.1200 /$ JCO.2007.12.9791

30. Hu H, Han H, Han XK, Wang WP, Ding H. Nomogram for Individualised Prediction of Liver Failure Risk After Hepatectomy in Patients With Resectable Hepatocellular Carcinoma: The Evidence From Ultrasound Data. Eur Radiol (2018) 28(2):877-85. doi: 10.1007/s00330-017-4900-2

31. Wang Y, Li J, Xia Y, Gong R, Wang K, Yan Z, et al. Prognostic Nomogram for Intrahepatic Cholangiocarcinoma After Partial Hepatectomy. J Clin Oncol (2013) 31(9):1188-95. doi: 10.1200/jco.2012.41.5984

32. Levy DA, Li H, Sterba KR, Hughes-Halbert C, Warren GW, Nussenbaum B, et al. Development and Validation of Nomograms for Predicting Delayed Postoperative Radiotherapy Initiation in Head and Neck Squamous Cell Carcinoma. JAMA Otolaryngol Head Neck Surg (2020) 146(5):455-64. doi: 10.1001/jamaoto.2020.0222

33. An C, Li X, Yu X, Cheng Z, Han Z, Liu F, et al. Nomogram Based on AlbuminBilirubin Grade to Predict Outcome of the Patients With Hepatitis C VirusRelated Hepatocellular Carcinoma After Microwave Ablation. Cancer Biol Med (2019) 16(4):797-810. doi: 10.20892/j.issn.2095-3941.2018.0486

34. Ni JY, Fang ZT, Sun HL, An C, Huang ZM, Zhang TQ, et al. A Nomogram to Predict Survival of Patients With Intermediate-Stage Hepatocellular Carcinoma After Transarterial Chemoembolization Combined With Microwave Ablation. Eur Radiol (2020) 30(4):2377-90. doi: 10.1007/s00330-019-06438-8

35. Huang Z, Gu Y, Zhang T, Wu S, Wang X, An C, et al. Nomograms to Predict Survival Outcomes After Microwave Ablation in Elderly Patients ( $>65$ Years Old) With Early-Stage Hepatocellular Carcinoma. Int J Hyperthermia (2020) 37(1):808-18. doi: 10.1080/02656736.2020.1785556

36. Kao WY, Su CW, Chiou YY, Chiu NC, Liu CA, Fang KC, et al. Hepatocellular Carcinoma: Nomograms Based on the Albumin-Bilirubin Grade to Assess the Outcomes of Radiofrequency Ablation. Radiology (2017) 285(2):670-80. doi: 10.1148/radiol.2017162382

37. Shi YF, Zhou P, Zhao YF, Liu WG, Tian SM, Liang YP. Microwave Ablation Compared With Laser Ablation for Treating Benign Thyroid Nodules in a Propensity-Score Matching Study. Front Endocrinol (Lausanne) (2019) 10: org/10.3389/fendo.2019.00874. doi: 10.3389/fendo.2019.00874

38. Ha EJ, Baek JH, Kim KW, Pyo J, Lee JH, Baek SH, et al. Comparative Efficacy of Radiofrequency and Laser Ablation for the Treatment of Benign Thyroid Nodules: Systematic Review Including Traditional Pooling and Bayesian Network MetaAnalysis. J Clin Endocrinol Metab (2015) 100(5):1903-11. doi: 10.1210/jc.2014-4077

39. Cesareo R, Manfrini S, Pasqualini V, Ambrogi C, Sanson G, Gallo A, et al. Laser Ablation Versus Radiofrequency Ablation for Thyroid Nodules: 12Month Results of a Randomized Trial (LARA II Study). J Clin Endocrinol Metab (2021) 106(6):1692-701. doi: 10.1210/clinem/dgab102

40. Cesareo R, Pacella CM, Pasqualini V, Campagna G, Iozzino M, Gallo A, et al. Laser Ablation Versus Radiofrequency Ablation for Benign Non-Functioning Thyroid Nodules: Six-Month Results of a Randomized, Parallel, Open-Label, Trial (LARA Trial). Thyroid (2020) 30(6):847-56. doi: 10.1089/thy.2019.0660

41. Deandrea M, Trimboli P, Mormile A, Cont AT, Milan L, Buffet C, et al. Determining an Energy Threshold for Optimal Volume Reduction of Benign 
Thyroid Nodules Treated by Radiofrequency Ablation. Eur Radiol (2021) 31 (7):5189-97. doi: 10.1007/s00330-020-07532-y

42. Trimboli P, Deandrea M. Treating Thyroid Nodules by Radiofrequency: Is the Delivered Energy Correlated With the Volume Reduction Rate? A Pilot Study. Endocrine (2020) 69(3):682-7. doi: 10.1007/s12020-020-02275-8

43. Zhang Y, Zhang MB, Luo YK, Li J, Zhang Y, Tang J. Effect of Chronic Lymphocytic Thyroiditis on the Efficacy and Safety of Ultrasound-Guided Radiofrequency Ablation for Papillary Thyroid Microcarcinoma. Cancer Med (2019) 8(12):5450-8. doi: 10.1002/cam4.2406

44. Sim JS, Baek JH. Long-Term Outcomes Following Thermal Ablation of Benign Thyroid Nodules as an Alternative to Surgery: The Importance of Controlling Regrowth. Endocrinol Metab (Seoul) (2019) 34(2):117-23. doi: 10.3803/EnM.2019.34.2.117

45. Yan L, Luo Y, Zhang M, Xiao J. Vital Volume Increase Versus Clinical Evaluation as the Indication of Additional Radiofrequency Ablation for Benign Thyroid Nodule: A Single Center Retrospective Study. Int J Hyperthermia (2020) 37(1):777-85. doi: 10.1080/02656736.2020.1778197

46. Park HS, Baek JH, Park AW, Chung SR, Choi YJ, Lee JH. Thyroid Radiofrequency Ablation: Updates on Innovative Devices and
Techniques. Korean J Radiol (2017) 18(4):615-23. . doi: 10.3348/kjr. 2017.18.4.615

Conflict of Interest: The authors declare that the research was conducted in the absence of any commercial or financial relationships that could be construed as a potential conflict of interest.

Publisher's Note: All claims expressed in this article are solely those of the authors and do not necessarily represent those of their affiliated organizations, or those of the publisher, the editors and the reviewers. Any product that may be evaluated in this article, or claim that may be made by its manufacturer, is not guaranteed or endorsed by the publisher.

Copyright (c) 2022 Yan, Zhang, Li, Li and Luo. This is an open-access article distributed under the terms of the Creative Commons Attribution License (CC BY). The use, distribution or reproduction in other forums is permitted, provided the original author(s) and the copyright owner(s) are credited and that the original publication in this journal is cited, in accordance with accepted academic practice. No use, distribution or reproduction is permitted which does not comply with these terms. 